\title{
New-onset QT prolongation is a novel predictor of mortality in critically ill patients
}

\author{
Yonghe Ding ${ }^{1 \dagger}$, Ryounghoon Jeon ${ }^{2 \dagger}$, Linyu Ran ${ }^{3}$, Wenzhi Pan ${ }^{4}$, Feilong Wang ${ }^{5,6^{*}}$ (D) and Qiang Li ${ }^{5^{*}}$
}

QT prolongation is associated with increased mortality in different types of patients $[1,2]$. QT prolongation is common in critically ill patients [3], and the association between heart rate corrected QT (QTc) and outcome in critically ill patients has raised broad interests most recently [4]. However, the prevalence of new-onset QT prolongation and its significance in these patients was not well studied yet.

Here, we prospectively recruited 505 consecutive ICU patients without known previous QT prolongation to evaluate the risk factors for new-onset QT prolongation and the prognostic value of QTc calculated by different methods. The baseline clinical and laboratory characteristics of subjects were shown in Table 1. The mean Bazett QT interval was $413.6 \pm$ $33.8 \mathrm{~ms}$. New-onset QT prolongation occurred in 99 patients (19.6\%). This occurrence is about 200-fold higher than that in the general population [5]. Intriguingly, the occurrence of nonthyroidal illness syndrome (NTIS) is significantly higher in patients with QT prolongation than those without (Table 1), indicating that NTIS might be a risk factor of QT prolongation. Indeed, multivariate linear regression showed that QTc was independently associated with NTIS, heart rate, level of serum potassium, gender, and estimated glomerular filtration rate (eGFR).
There was a significantly graded increase in mortality rate across increasing QTc quintile $(p=0.004)$ (Fig. 1a). The overall mortality rate in patients with a new-onset QTc prolongation is more than two times higher than those patients without $(22.2 \%$ vs 9.6, $\mathrm{OR}=2.69, p=0.001$ ) (Fig. 1b). Multivariate logistic regression showed that QT prolongation is still independently associated with ICU mortality even after adjusted for age and gender $(p=0.001,95 \%$ C.I., 1.51-4.79). However, QT prolongation is no longer a predictor of ICU mortality if APACHE-II score was further adjusted $(p=0.329)$, likely due to that QTc itself is strongly associated with APACHE-II score $(r=-0.235, p<0.001)$.

As Bazett's formula can over-correct QT at high heart rates and under-correct it at low heart rates, we then evaluated the prognostic value of QTc calculated using additional formulas including Fridericia's, Framingham's, and Hodges's. We found that patients in quintile 5 have significantly higher mortality than patients in the combination of quintiles 1-4 regardless of which formula was used (all $p<0.05)$.

In summary, QT prolongation determined by baseline ECG can serve as a novel indicator of the severity of illness in critically ill patients. NTIS is a new risk factor of QT prolongation in critically ill patients.

\footnotetext{
* Correspondence: dr.feilongwang@gmail.com; liqressh@hotmail.com

†Yonghe Ding and Ryounghoon Jeon contributed equally to this work.

${ }^{5}$ Department of Pulmonary and Critical Care Medicine, Shanghai East

Hospital, Tongji University, Shanghai 200120, China

Full list of author information is available at the end of the article
} 
Table 1 Clinical and laboratory characteristics of subjects

\begin{tabular}{|c|c|c|c|c|}
\hline & All & $\begin{array}{l}\text { Normal QT } \\
(N=406)\end{array}$ & $\begin{array}{l}\text { QT prolongation } \\
(N=99)\end{array}$ & $p$ \\
\hline Age (years) & $63.7 \pm 18.2$ & $62.8 \pm 18.5$ & $67.4 \pm 16.6$ & 0.20 \\
\hline Male (\%) & $305(60.4)$ & $237(58.4)$ & $68(68.7)$ & 0.06 \\
\hline Heart rate (BPM) & $85.2 \pm 20.4$ & $83.7 \pm 20.8$ & $91.3 \pm 17.5$ & 0.01 \\
\hline Positive cTNT (\%) & 52.5 & 46.5 & 76.8 & $<0.001$ \\
\hline LogNT-proBNP & $2.3 \pm 0.7$ & $2.2 \pm 0.7$ & $2.6 \pm 0.6$ & 0.91 \\
\hline $\mathrm{Na}+(\mathrm{mmol} / \mathrm{L})$ & $140.2 \pm 5.7$ & $140.0 \pm 5.5$ & $141.0 \pm 6.5$ & 0.15 \\
\hline $\mathrm{K}+(\mathrm{mmol} / \mathrm{L})$ & $3.9 \pm 0.6$ & $3.9 \pm 0.6$ & $3.8 \pm 0.7$ & 0.10 \\
\hline $\mathrm{Cl}-(\mathrm{mmol} / \mathrm{L})$ & $104.7 \pm 6.4$ & $104.6 \pm 6.0$ & $105.5 \pm 7.8$ & 0.04 \\
\hline $\mathrm{Ca} 2+(\mathrm{mmol} / \mathrm{L})$ & $2.08 \pm 0.21$ & $2.10 \pm 0.19$ & $2.02 \pm 0.25$ & 0.001 \\
\hline FBG $(\mathrm{mmol} / \mathrm{L})$ & $7.53 \pm 3.28$ & $7.36 \pm 3.02$ & $8.26 \pm 4.15$ & 0.02 \\
\hline $\mathrm{eGFR}\left(\mathrm{mL} / \mathrm{min} / 1.73 \mathrm{~m}^{2}\right)$ & $86.7 \pm 44.1$ & $91.6 \pm 42.9$ & $66.3 \pm 43.5$ & 0.49 \\
\hline CKD grade ${ }^{\#}$ & $1.95 \pm 1.13$ & $1.80 \pm 1.01$ & $2.56 \pm 1.35$ & $<0.001$ \\
\hline APACHE- II (points) & $15.0 \pm 8.4$ & $14.2 \pm 8.0$ & $18.7 \pm 9.2$ & 0.006 \\
\hline TT3 (nmol/L) & $0.92 \pm 0.45$ & $0.96 \pm 0.48$ & $0.73 \pm 0.25$ & 0.004 \\
\hline TT4 (nmol/L) & $86.4 \pm 30.1$ & $88.7 \pm 30.9$ & $77.4 \pm 24.8$ & 0.19 \\
\hline FT3 (pmol/L) & $3.44 \pm 1.10$ & $3.52 \pm 1.18$ & $3.15 \pm 0.59$ & 0.17 \\
\hline FT4 (pmol/L) & $15.5 \pm 4.8$ & $15.5 \pm 5.1$ & $15.4 \pm 3.6$ & 0.67 \\
\hline $\mathrm{TSH}(\mathrm{IU} / \mathrm{mL})$ & $1.34 \pm 1.35$ & $1.34 \pm 1.28$ & $1.32 \pm 1.61$ & 0.06 \\
\hline \#NTIS (\%) & 59.3 & 55.0 & 76.3 & $<0.001$ \\
\hline
\end{tabular}

BPM beats per minute, FBG fasting blood glucose, eGFR estimated glomerular filtration rate, CKD chronic kidney disease, APACHE // score Acute Physiology and Chronic Health Evaluation II score. TT3 total triiodothyronine, TT4 total thyroxine, FT3 free triiodothyronine, FT4 free thyroxine, TSH thyroid-stimulating-hormone, rT3 reverse triiodothyronine, NTIS nonthyroidal illness syndrome

\#NTIS: Euthyroid patients with fT3 decreased below the normal range $(<3.5 \mathrm{pmol} / \mathrm{L})$ during critical illness

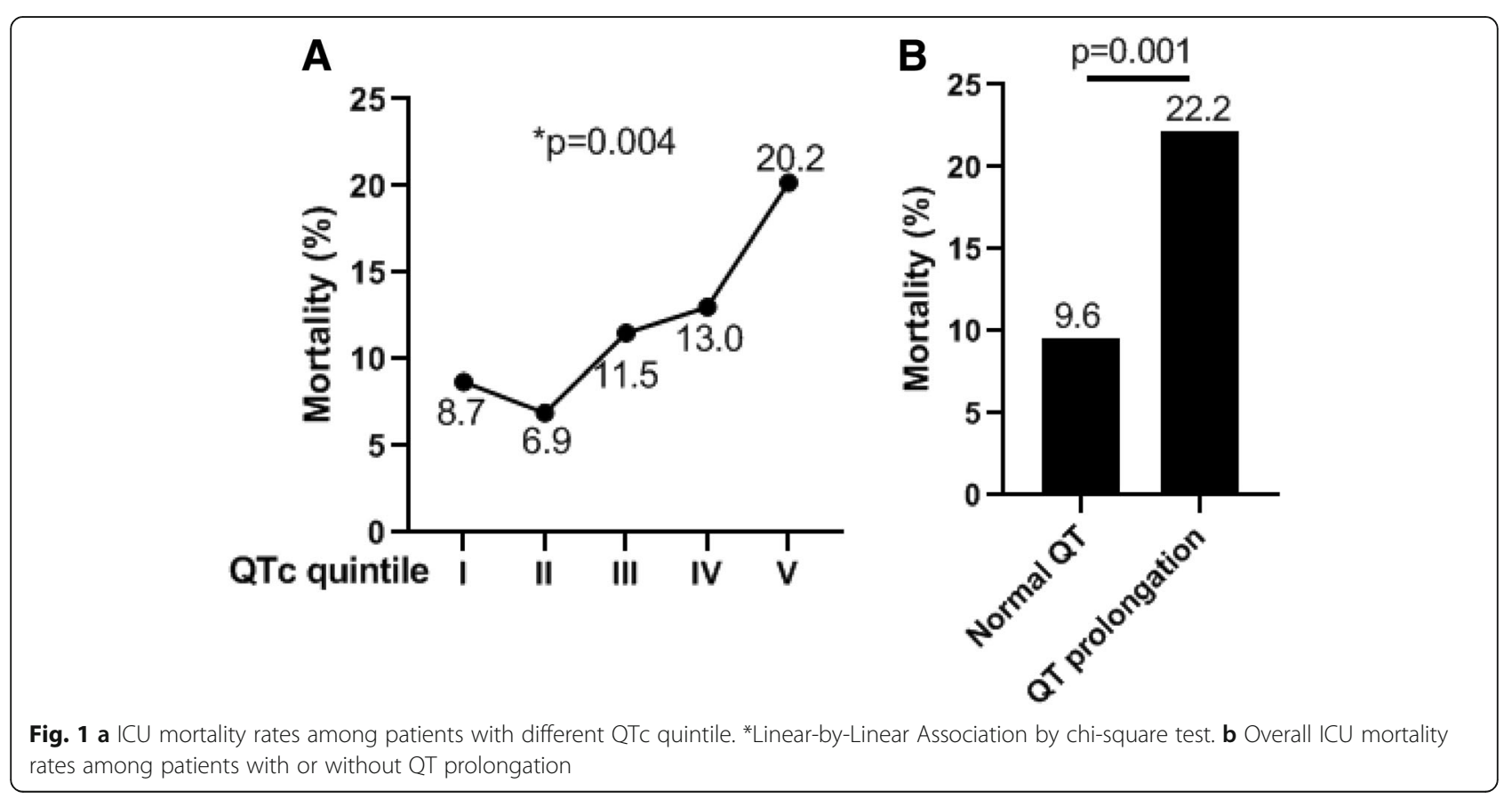




\section{Abbreviations}

APACHE-II score: Acute Physiology and Chronic Health Evaluation II score; eGFR: Estimated glomerular filtration rate; ICU: Intensive care unit; NTIS: Nonthyroidal illness syndrome; QTc: Heart rate-corrected QT

\section{Acknowledgements}

None

\section{Authors' contributions}

FW conceived and designed the study. YD, RJ, LR, and WP contributed to data acquisition and analysis. QL interpreted the data and provided insightful input to this study. All authors read and approved the final manuscript.

\section{Funding}

This work was supported by the "Gaoyuan" project from Pudong Health and Family Planning Commission (PWYgy2018-06).

\section{Availability of data and materials}

The datasets used and/or analyzed during the current study are available from the corresponding author on reasonable request.

\section{Ethics approval and consent to participate}

The study was approved by the Shanghai Jiaotong University Xinhua Hospital Ethics Committee (XHEC2011-002).

\section{Consent for publication}

Not applicable

\section{Competing interests}

The authors declare that they have no competing interests.

\section{Author details}

${ }^{1}$ The Affiliated Hospital of Qingdao University, The Biomedical Sciences Institute of Qingdao University, Qingdao University, Qingdao 266003, China. 2Department of Cardiovascular Medicine, Mayo Clinic, Rochester, MN 55902, USA. ${ }^{3}$ Medical College, Tongji University, Shanghai 200092, China. ${ }^{4}$ Department of Cardiology, Shanghai Institute of Cardiovascular Disease, Zhongshan Hospital, Fudan University, Shanghai 200032, China. ${ }^{5}$ Department of Pulmonary and Critical Care Medicine, Shanghai East Hospital, Tongji University, Shanghai 200120, China. ${ }^{6}$ Department of Emergency, Xinhua Hospital Affiliated to Shanghai Jiaotong University School of Medicine, Shanghai 200092, China.

Received: 10 May 2019 Accepted: 11 June 2019

Published online: 21 June 2019

\section{References}

1. O'Neal WT, Singleton MJ, Roberts JD, Tereshchenko LG, Sotoodehnia N, Chen LY, Marcus GM, Soliman EZ. Association between QT-interval components and sudden cardiac death: the ARIC study (atherosclerosis risk in communities). Circ Arrhythm Electrophysiol. 2017;10:e005485.

2. Zhang Y, Post WS, Dalal D, Blasco-Colmenares E, Tomaselli GF, Guallar E. QTinterval duration and mortality rate: results from the Third National Health and Nutrition Examination Survey. Arch Intern Med. 2011;171:1727-33.

3. Fukui $\mathrm{S}$, Katoh $\mathrm{H}$, Tsuzuki $\mathrm{N}$, Ishihara $\mathrm{S}$, Otani $\mathrm{N}$, Ooigawa $\mathrm{H}$, Toyooka $\mathrm{T}$, Ohnuki A, Miyazawa T, Nawashiro H, Shima K. Multivariate analysis of risk factors for QT prolongation following subarachnoid hemorrhage. Crit Care. 2003;7:R7.

4. Javanainen T, Ishihara S, Gayat E, Charbit B, Jurkko R, Cinotti R, Mebazaa A. Prolonged corrected QT interval is associated with short-term and longterm mortality in critically ill patients: results from the FROG-ICU study. Intensive Care Med. 2019;11:1-3.

5. van Noord C, Eijgelsheim M, Stricker BH. Drug- and non-drug-associated QT interval prolongation. Br J Clin Pharmacol. 2010;70:16-23.

\section{Publisher's Note}

Springer Nature remains neutral with regard to jurisdictional claims in published maps and institutional affiliations. 